\title{
THE GENERAL SOLUTION TO A SYSTEM OF ADJOINTABLE OPERATOR EQUATIONS OVER HILBERT $C^{*}$-MODULES
}

\author{
QING-WEN WANG, CHANG-ZHOU DONG
}

\begin{abstract}
We establish necessary and sufficient conditions for the existence of solution to the system of adjointable operator equations $A_{1} X=D_{1}, X B_{2}=D_{2}, A_{3} X B_{3}+B_{3}^{*} X^{*} C_{3}=D_{3}$ over the Hilbert $C^{*}$-modules. We also give the explicit expression of the general solution to this system when the solvability conditions are satisfied. As an application, we investigate the anti-reflexive Hermitian solution to the system of complex matrix equations $A X=B, X C=D, E X E^{*}=F$. The findings of this paper extend some known results in the literature.
\end{abstract}

Mathematics subject classification (2010): 47A62, 46L08, 15A24, 47A52.

Keywords and phrases: Hilbert $C^{*}$-modules, operator equation, system of operator equations, MoorePenrose inverse.

\section{REFERENCES}

[1] D. S. DJordjević, Explicit solution of the operator equation $A^{*} X+X^{*} A=B$, J. Comput. Appl. Math., 200 (2007), 701-704.

[2] D. S. CvetKović-ILIĆ, The solutions of some operator equations, J. Korean Math. Soc., 45(5) (2008), 1417-1425.

[3] Q. XU, L. Sheng, Y. Gu, The solutions to some operator equations, Linear Algebra Appl., 429 (2008), 1997-2024.

[4] Q. XU, Common Hermitian and positive solutions to the adjointable operator equations $A X=C$, $X B=D$, Linear Algebra Appl., 429 (2008), 1-11.

[5] A. DajiĆ, J. J. Koliha, Positive solutions to the equations $A X=C$ and $X B=D$ for Hilbert space operators, J. Math. Anal. Appl., 333 (2007), 567-576.

[6] A. Dajić, J. J. Koliha, Equations $a x=c$ and $x b=d$ in rings and rings with involution with applications to Hilbert space operators, Linear Algebra Appl., 429 (2008), 1779-1809.

[7] X. FANG, J. YU, H. YAO, Solutions to operator equations on Hilbert $C^{*}$-modules, Linear Algebra Appl., 431 (2009), 2142-2153.

[8] H. C. CHEN, Generalized reflexive matrices: special properties and applications, SIAM J. Matrix Appl., 19 (1998), 140-153.

[9] Z. Y. PENG, X. Y. HU, The reflexive and anti-reflexive solutions of matrix equation $A X=B$, , Linear Algebra Appl., 375 (2003), 147-155.

[10] H. X. ChANG, Q. W. WANG, Reflexive solutions to a system of matrix equations, Journal of Shanghai University (English edition), 11 (2007), 355-358.

[11] Q. W. WANG, F. ZHANG, The reflexive re-nonnegative definite solution to a quaternion matrix equation, Electron. J. Linear Algebra, 17 (2008), 88-101.

[12] I. KAPLANSKY, Modules over operator algebras, Amer J. Math., 75 (1953), 839-858.

[13] N. E. WegGe-Olsen, $K$-theory and $C^{*}$-algebras-a friendly approach, Oxford University Press, Oxford, England, 1993.

[14] E. C. LANCE, Hilbert $C^{*}$-modules-A Toolkit for Operator Algebraists, Cambridge University Press, 1995.

[15] S. L. Campbell, C. D. Meyer JR., Generalized inverses of linear transformations, Dover Publication, Inc., New York, 1991.

[16] J. C. Hou, J. L. CuI, Theorem of linear mapping on operator algebras, Science Press, BeiJing, 2002. 
[17] A. Ben-Israel, T. N. E. Greville, Generalized inverse: theory and applications, Second Ed., Springer, 2003.

[18] G. R. WANG, Generalized Inverse: Theory and Computations, Science Press, BeiJing, 2004. 